\title{
Talleres de alfabetización digital surgidos a partir del programa UNAMITA ¡Ciérrale a la brecha digital!
}

DOI: $10.46932 / \mathrm{sfjdv} 2 \mathrm{n} 2-128$

Received in: March 1st, 2021

Accepted in: May 30th, 2021

\author{
Mtra. Mara Nieto Hernández \\ Facultad de Filosofía y Letras \\ Universidad Nacional Autónoma de México \\ Circuito Interior, Ciudad Universitaria, s/n, C.P. 04510, Ciudad de México. \\ E-mail: ia.maranieto@gmail.com \\ Lic. Blanca Delia De la Rosa Vergara \\ Facultad de Filosofía y Letras \\ Universidad Nacional Autónoma de México \\ Circuito Interior, Ciudad Universitaria, s/n, C.P. 04510, Ciudad de México. \\ E-mail: deliadlr81@gmail.com
}

\section{RESUMEN}

En el presente artículo se abordan dos experiencias de trabajo que surgieron a partir del programa UNAMITA ¡Ciérrale a la Brecha Digital! perteneciente a la Universidad Nacional Autónoma de México, el cual se centra en el desarrollo de habilidades educativas y sociales para el manejo correcto de las herramientas digitales. La primera experiencia de trabajo consiste en un taller de alfabetización digital llamado "Docentes apropiándose de las tecnologías digitales". Este taller estuvo dirigido a docentes voluntarios que laboran en una asociación civil que se encuentra dentro de una comunidad marginada ubicada en Tultitlán, Estado de México. La segunda experiencia de trabajo corresponde a un taller de alfabetización digital llamado "El que busca encuentra". Este taller estuvo dirigido a alumnos de educación básica del "Colegio Pilcalli" ubicado en la comunidad de San Miguel Tlaixpan, en el municipio de Texcoco, en el Estado de México. Ambas experiencias están enfocadas en el desarrollo de competencias digitales y en el cierre de la brecha digital. En el artículo se presenta de manera general la metodología que se utilizó en cada una de las experiencias de trabajo, así como los resultados obtenidos tras su implementación.

Palabras clave: Alfabetización digital, competencias digitales, brecha digital.

\section{INTRODUCCIÓN}

Los diferentes cambios que se van presentando de manera global dentro de la sociedad han abierto nuevas formas de crecimiento a partir de las tecnologías, debido a que a través de ellas, se permite la búsqueda de mejores oportunidades para aprender lo que se quiere aprender, para generar nuevos conocimientos y para construir experiencias que funjan como un vehículo para innovar (Santana et al., 2021). Sin embargo, esos cambios globales también han dado lugar a una denominada brecha digital, debido a que por una u otra causa, no todas las personas han podido beneficiarse de las nuevas 
oportunidades, puesto que no han tenido un acercamiento a la información, al conocimiento y a la educación mediante las nuevas tecnologías (Cabero, 2004).

El término de brecha digital se genera a través de las diversas situaciones que se presentan de acuerdo al resultado de diferencias de acceso, disponibilidad de infraestructura y a la comunicación; estos aspectos influyen de alguna forma en el desarrollo que se presenta en el contexto social y cultural, así como económico del país (Gros y Contreras, 2006). Debido a lo anterior, puede decirse que, una de las principales desventajas de la brecha digital consiste en que poco a poco "esta se está convirtiendo en un instrumento de separación, marginación y exclusión", debido a que quienes no tienen acceso a las tecnologías cada vez tienen menos oportunidades para sobresalir dentro de la nueva sociedad (Cabero, 2004).

De acuerdo con diversos autores, se sabe que, una herramienta útil para cerrar la brecha digital y lograr un manejo correcto de las tecnologías en la educación consiste en la alfabetización digital (Gros y Contreras, 2006; Gutiérrez, 2008). Cuando comenzamos con nuestra educación, aprendemos a leer, aprendemos a escribir, conocemos por primera vez los números y la infinidad de oportunidades que estos tienen. Con la llegada de las tecnologías, no es solo conocer las letras y saber cómo formarlas, pues los textos están en las pantallas y reconocer cuáles son los correctos, así como los de calidad, forma parte de la alfabetización [digital] que debemos de tener.

Ante la situación de brecha digital que se presenta sobre todo en los diferentes campos educativos, se han creado diferentes programas para dar cierre a la misma. Uno de esos programas es UNAMITA ¡Ciérrale a la Brecha Digital!, programa que se encarga de promover información relacionada con las oportunidades que brinda la tecnología, no solo en la educación, sino en los diferentes enfoques de la sociedad, además de ofrecer propuestas -como la alfabetización digital-para dar término a la separación entre la llegada de las tecnologías y el uso correcto de estas.

A partir de esta búsqueda de cierre de la brecha digital y con apoyo del programa UNAMITA ¡Ciérrale a la brecha digital! es como surgieron las propuestas de las autoras del presente artículo. En dichas propuestas se buscó alfabetizar digitalmente a los docentes voluntarios de una asociación civil localizada el municipio de Tultitlán, así como orientar a los alumnos de la escuela secundaria "Colegio Pilcalli” en la búsqueda de información de calidad.

\section{LA ALFABETIZACIÓN DIGITAL}

La existencia de la brecha digital ha cambiado drásticamente la manera de alfabetizar a las generaciones. Hoy en día, ya no basta con que un ciudadano sepa leer, escribir y algo de aritmética. En estos tiempos, es necesario también poseer un dominio apropiado de las tecnologías, por lo que además 
de la alfabetización convencional, se trabaja también con una alfabetización digital (Semenov, Pereversev y Bulin-Socolova, 2005).

Un gran número de autores se ha enfocado en definir a la alfabetización digital. Para Gros y Contreras (2006), la alfabetización digital además de aludir al manejo técnico de los ordenadores, también "se refiere a las capacidades para identificar y evaluar la información utilizando cualquier herramienta que se considere apropiada -como las proporcionadas por las TIC-y aprender a leer la información dentro de este contexto sociocultural" (p. 108).

Por su parte, Fresno (2007) considera que la alfabetización digital consiste en un proceso en el que se adquieren las siguientes habilidades: "conocimiento de los sistemas informáticos, uso del sistema operativo, búsqueda y selección de información en Internet, comunicación personal y trabajo colaborativo en redes, procesamiento de textos, tratamiento de la imagen, utilización de hoja de cálculo, entretenimiento y aprendizaje con las TIC, uso de bases de datos y actitudes generales ante las TIC" (p.4).

De acuerdo con Area y Pessoa, la alfabetización digital abarca la adquisición de competencias digitales, las cuales han sido divididas en las siguientes dimensiones:

- Competencias instrumentales: se refieren al dominio técnico de la tecnología, es decir, a la adquisición del conocimiento teórico y práctico para el manejo del hardware y del software.

- Competencias cognitivo-intelectuales: consisten en el conjunto de conocimientos y habilidades para buscar, seleccionar, analizar, interpretar y recrear la gran cantidad de información a la que se tiene acceso a partir de las tecnologías digitales y dentro de la Sociedad de la Información.

- Competencias socio-comunicacionales: aluden a las habilidades relacionadas con la creación y difusión de textos de distinta naturaleza, utilizando diversos tipos de lenguaje.

- Competencias axiológicas: se refieren a la toma de conciencia sobre el papel cultural, político, ético y democrático de las tecnologías y de la información que proviene de ellas.

- Competencias emocionales: consisten en la habilidad para controlar las emociones negativas y desarrollar la empatía, al momento de utilizar las tecnologías o los ambientes virtuales (Area y Pessoa, 2012).

Por su parte, para Semenov et al. (2005), el proceso de alfabetización digital involucra básicamente los siguientes tres pasos: búsqueda de información a partir de las tecnologías, comunicación en ambientes virtuales y diseño de objetos y acciones digitales. Los pasos anteriores se relacionan con el proceso de alfabetización tradicional (ver figura 1), debido a que buscar información equivale a aprender a leer, la comunicación en ambientes virtuales se equipara con aprender a escribir, y finalmente, el diseño de objetos y acciones se relaciona con aprender a hacer cálculos básicos (p.25). 
Figura 1. Diagrama que compara el proceso de alfabetización tradicional con la alfabetización digital.

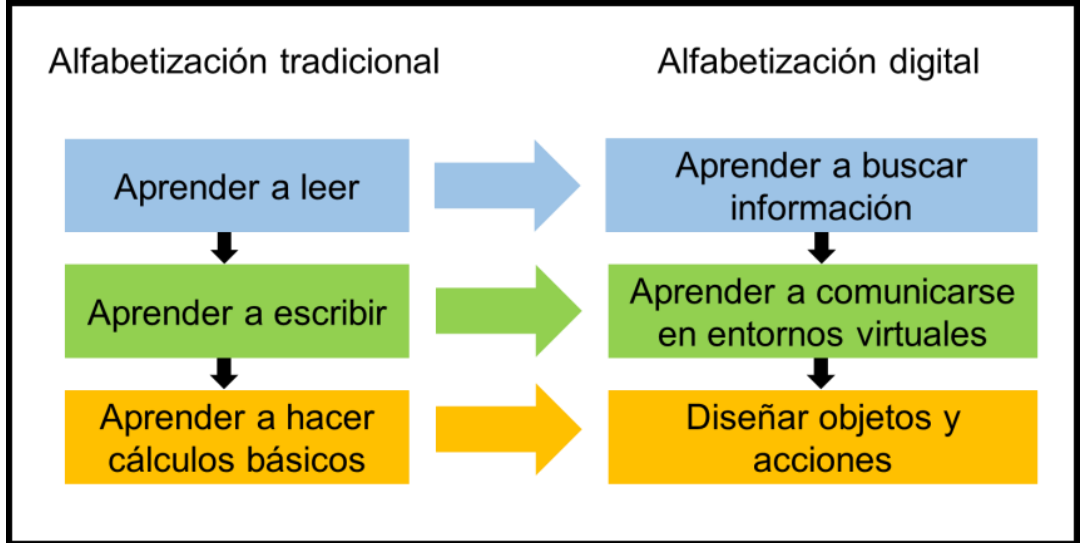

El tema de la alfabetización digital ha sido abordado dentro de intervenciones realizadas a partir del programa UNAMITA ¡Ciérrale a la brecha digital! El programa UNAMITA ¡Ciérrale a la brecha digital! es un programa de formación de tutores en ciudadanía y tecnologías digitales, del Departamento de Servicio Social de la Facultad de Filosofía y Letras de la UNAM, que cuenta con apoyo de PAPIME PE404119. El objetivo general de UNAMITA ¡Ciérrale a la brecha digital! consiste en "ofrecer a los estudiantes acciones formativas dirigidas al desarrollo de habilidades técnicas, sociales y éticas relativas al uso de las TIC, y que con ello contribuyan en otros en su construcción de su ciudadanía digital". A continuación se describirán dos de los talleres de alfabetización digital que han surgido a partir del programa UNAMITA ¡Ciérrale a la brecha digital!

\section{TALLER DE ALFABETIZACIÓN DIGITAL NO.1: “DOCENTES APROPIÁNDOSE DE LAS TECNOLOGÍAS DIGITALES" \\ El taller de alfabetización digital "Docentes apropiándose de las tecnologías digitales" abarcó los} tres pasos del proceso de alfabetización digital sugerido por Semenov et al., es decir, se enfocó en instruir a los docentes en torno a la búsqueda de información a partir de las tecnologías, la comunicación en ambientes virtuales y el diseño de objetos y acciones digitales. De igual forma, este taller pretendió desarrollar en los asistentes, competencias digitales pertenecientes a todas y cada una de las cinco dimensiones sugeridas por Area y Pessoa.

El taller de alfabetización digital se desarrolló en una asociación civil que se encuentra dentro de una comunidad marginada del municipio de Tultitlán, en el Estado de México. Esta intervención estuvo dirigida a docentes voluntarios que prestan sus servicios de forma desinteresada a la asociación civil. Una de las principales metas que tiene la asociación civil, en conjunto con los docentes voluntarios a quienes se les impartió el taller de alfabetización digital, consiste en brindar educación no formal (regularización, preparación para el empleo, acompañamiento) a los miembros de la comunidad, con la finalidad de que estos tengan otras perspectivas de vida en lo que concierne a los ámbitos laboral y económico. 
El taller de alfabetización digital se propuso, debido a que se observó que aunque los docentes voluntarios tenían a su alcance un laboratorio de cómputo con Internet, rara vez hacían uso de las tecnologías para impactar e innovar su práctica educativa, ya que no contaban con una apropiación significativa de los recursos a los que tenían acceso. Lo anterior, sin duda constituía la existencia de una brecha digital en los docentes voluntarios que prestan sus servicios para la asociación civil.

El taller de alfabetización digital constó de tres etapas:

- La primera etapa estuvo conformada por sesiones presenciales, en las cuales se abordaron las siguientes temáticas: búsqueda de información en la Web, uso de blogs y bibliotecas digitales, realización de reportes de investigación, comunicación asertiva en ambientes virtuales, redes sociales aplicadas a educación, creación de materiales didácticos (infografías y presentaciones digitales) y acceso a sistemas de educación no formal en línea (MOOC'S o cursos abiertos masivos en línea).

- Durante la segunda etapa, los docentes voluntarios exploraron las opciones educativas disponibles a partir de MOOC'S y estudiaron un curso que complementó sus conocimientos en torno a contenidos o competencias docentes, de acuerdo a las necesidades personales de cada uno.

- La tercera etapa del taller de alfabetización digital consistió en una práctica guiada, en la cual los docentes hicieron uso del conocimiento adquirido durante las dos etapas anteriores. En esta práctica guiada, los asistentes planearon y realizaron una intervención, en donde ocuparon información adquirida a partir del MOOC cursado. Los docentes voluntarios utilizaron ambientes virtuales para comunicarse con sus estudiantes, y diseñaron acciones digitales en su intervención docente.

Para evaluar el alcance del taller de alfabetización digital, se calculó el nivel grupal de alfabetización digital (\%), antes y después de la intervención. Este parámetro se determinó a partir de un instrumento estandarizado propuesto por Rangel y Peñalosa (2013), el cual ocupa como herramienta la aplicación de un cuestionario de autoevaluación en donde se califica la apropiación de competencias digitales. De manera general puede decirse que, el nivel grupal de alfabetización digital (\%) en los docentes voluntarios pasó de un nivel bajo a un nivel medio, debido a que incrementó de un $32.11 \%$ a un 59.8\%. A partir de los resultados obtenidos, se sabe que las competencias digitales que tuvieron un mayor desarrollo fueron las competencias cognitivo-intelectuales, las competencias socio comunicacionales y las competencias axiológicas, sin embargo, también las competencias instrumentales y las competencias emocionales se vieron beneficiadas. 
Con respecto al estudio de los MOOC'S, los docentes voluntarios han comentado que, a partir de la intervención, ellos ahora cuentan con herramientas para desarrollar una práctica docente más apegada a las necesidades reales de la comunidad, debido a que tienen la posibilidad de cursar MOOC'S relacionados con cualquier temática y a través de estos crear el contenido de nuevas y más significativas intervenciones. Es debido a lo anterior que, varios de los docentes voluntarios que tomaron el taller han seguido cursando MOOC'S, terminando al menos uno por mes.

La intervención en la comunidad realizada por parte de los docentes voluntarios como producto de la tercera etapa consistió en la impartición de un curso denominado "Autoestima y manejo de emociones" y dirigido a los padres de familia que asisten de forma regular a la asociación civil. Los docentes voluntarios utilizaron los MOOC'S cursados como fuentes de consulta para desarrollar el contenido del curso. Durante la tercera etapa se percibió que los docentes voluntarios dominaban los temas aprendidos en los MOOC'S, y que incluso, los complementaban con sus propias experiencias. Las tecnologías digitales utilizadas durante el curso "Autoestima y manejo de emociones" fueron las siguientes: grupos de difusión a partir de la red social WhatsApp para una constante comunicación entre docentes voluntarios y participantes, infografías como material didáctico y presentaciones como material de apoyo.

Si bien es cierto que, el taller de alfabetización digital no representa la solución absoluta a los problemas de marginación que existen en la comunidad, este taller funge como una herramienta para cambiar el panorama, primeramente en los docentes voluntarios de la asociación civil y posteriormente en los habitantes de la comunidad. Lo anterior se sustenta con la siguiente tesis de Gutiérrez (2008): "Si hay que preparar a los niños y jóvenes para vivir en la Sociedad de la Información, habrá que capacitar a sus maestros y profesores en este sentido, y eso, no se consigue simplemente comprando ordenadores y enseñándoles su manejo para entrar en Internet." (p. 205).

Partiendo de la base del diagnóstico de una brecha digital en los docentes voluntarios, debido a que en un principio, ellos mismos confesaron no tener conocimientos básicos para implementar las tecnologías en su labor cotidiana, puede decirse que el taller de alfabetización digital pudo servir de preámbulo para que los mismos docentes voluntarios consideraran tener un mayor acercamiento a las tecnologías digitales, ya que hoy en día, después del taller, los docentes voluntarios tienen competencias para buscar información en la Web, conocen la forma de comunicarse efectivamente dentro de entornos virtuales, saben cómo diseñar algunas herramientas digitales para mejorar sus intervenciones educativas y han tenido la experiencia de cursar MOOC'S para complementar sus conocimientos docentes.

A manera de cierre general del taller de alfabetización digital "Docentes apropiándose de las tecnologías digitales" se puede comentar lo siguiente: 
- Hoy, a corto plazo, los docentes voluntarios están conscientes que, las competencias digitales que ellos han adquirido constituyen una primera fase para construir el conocimiento y generar nuevas estrategias en su práctica.

- A mediano plazo se pretende que, los docentes voluntarios motiven a la comunidad a aprender sobre las nuevas tecnologías y su utilización.

- A largo plazo se pretende que, valiéndose de la motivación por la tecnología, los docentes voluntarios ayuden a los habitantes de la comunidad marginada a desarrollar competencias digitales que les permitan participar activamente en las nuevas sociedades, y que de esta forma, se busque la mejora de la calidad de vida dentro de la comunidad.

\section{TALLER DE ALFABETIZACIÓN DIGITAL NO.2: "EL QUE BUSCA ENCUENTRA"}

La intervención educativa llamada "El que busca encuentra" consistió en un taller de orientación digital, enfocado en el primer paso del proceso de alfabetización digital -búsqueda de información-, y pretendió desarrollar básicamente competencias digitales de tipo cognitivo-intelectuales.

El taller estuvo dirigido a alumnos de secundaria de la comunidad de San Miguel Tlaixpan, del municipio de Texcoco, en el Estado de México. A través de diferentes encuestas realizadas a los alumnos, así como a partir de observaciones de campo, se detectó que antes de la implementación del taller, la mayoría de los estudiantes, al requerir algún tipo de información, no solo educativa sino también de su vida diaria, recurrían a los principales buscadores Web, dando como resultado que ellos optaran por tomar las primeras opciones de búsqueda, sin detenerse a observar qué tan válida y de qué tanta calidad era la información que ellos consultaban.

El objetivo principal del taller fue orientar a los alumnos sobre las diferentes oportunidades de búsqueda que pueden obtener a partir de los buscadores, así como las diferentes herramientas que la Web brinda. Siendo adolescentes ellos prefieren la opción más dinámica y atractiva, debido a lo anterior, los videos e imágenes son los recursos principales para sus investigaciones. Es por ello que, brindar oportunidades de búsqueda y animarlos a analizar críticamente toda la información que llega a sus manos, es indispensable.

Durante el diseño del taller de alfabetización digital "El que busca encuentra" fue importante tomar en cuenta el concepto de Sociedad del Conocimiento, el cual "hace especialmente hincapié en la capacidad para producir e integrar nuevos conocimientos y acceder a la información, el conocimiento, los datos y una vasta gama de conocimientos prácticos” (UNESCO, 2005, p.230).

Con base al concepto de Sociedad del Conocimiento, fue esencial tomar en cuenta las capacidades que tienen los alumnos de secundaria, debido a que a partir de estas, se buscó lograr que durante el taller 
los alumnos fueran capaces de llevar a cabo la conexión pertinente entre información y conocimiento. Se tuvo como base la siguiente tesis que propone Barceló (2001) “quien posee únicamente la información domina el saber qué y un saber por qué mientras que quien posee conocimientos domina un saber cómo y un saber quién" (p.14).

El taller de alfabetización digital "El que busca encuentra" se impartió en cuatro sesiones, en las cuales se brindaron conceptos oportunos para la apropiación de habilidades pertinentes, útiles para el desarrollo tecnológico que se aplica en la educación de los estudiantes. Las temáticas abordadas durante esas cuatro sesiones se describen a continuación:

- Sesión 1. Ciudadanía digital y brecha digital: En esta sesión se buscó crear el conocimiento necesario en los alumnos, para que ellos identificaran los comportamientos y la importancia de su crecimiento en el campo digital.

- Sesión 2. Habilidades y necesidades: Los alumnos identificaron cuáles son aquellos campos en donde ellos se consideran hábiles, posteriormente conocieron cuáles son sus necesidades inmediatas y finalmente descubrieron cómo pueden cubrir esas necesidades utilizando las habilidades con las que ya cuentan. Además aprendieron cómo cubrir sus necesidades en el campo educativo utilizando las habilidades que ya tienen.

- Sesión 3. Los buscadores Web: Durante esta sesión, los estudiantes conocieron cuáles son todos los buscadores a los que pueden recurrir para una búsqueda de información y los beneficios que estos les brindan. Además aprendieron cómo generar una opinión crítica acerca de aquellos resultados que se obtienen, y a partir de ello lograron evaluar la validez de la información.

- Sesión 4. Compartir y conocer resultados: En esta sesión, los alumnos reflexionaron sobre la importancia de utilizar correctamente las opciones aprendidas durante las sesiones anteriores. De igual forma, se dieron cuenta de que pueden compartir los conocimientos adquiridos durante el taller y ayudar a alguien más a recurrir a buscadores de información de calidad.

Para la evaluación de dicho taller se optó por la recopilación de actividades, elaborando de esta forma un portafolio de evidencias. De acuerdo con Barberá (2006) un portafolio es una herramienta de evaluación donde la enseñanza de aprendizaje funciona de apoyo, tanto para el alumno como para el docente. Un portafolio permite demostrar lo que se está aprendiendo, a la vez que posibilita al profesor dar un seguimiento del progreso de este aprendizaje. Cada evidencia se acompaña por una justificación por parte del alumno, con ello se reafirma la opinión crítica sobre el propio resultado y se evalúa aquello que se pretende aprender y cómo se está desarrollando, lo que ayuda a la evaluación continua y coherente del portafolio. 
Durante las diferentes actividades, búsquedas y justificaciones que se recabaron en el portafolio se pudo observar un gran progreso en los alumnos que tomaron el taller, ya que se detectó que los alumnos lograron incrementar su pensamiento crítico sobre las diferentes plataformas de búsqueda. Con ayuda del portafolio y la constante autoevaluación, los alumnos lograron utilizar plataformas educativas con mayor productividad, así como plataformas de video, revistas y bibliotecas académicas.

Para dar un cierre general del taller "El que busca encuentra", se puede comentar lo siguiente:

- Al día de hoy, los alumnos reconocen la importancia de la búsqueda de información y la opinión crítica que ellos deben de formular durante sus búsquedas.

- Quienes tomaron el taller entienden la importancia de una alfabetización digital y de conocer los conceptos de ciudadanía digital en su entorno como adolescentes, para actuar sobre aquellos comportamientos que forman parte de su educación.

\section{CONCLUSIONES}

De manera global, se puede mencionar que los talleres de alfabetización digital constituyen una forma de apoyar al cierre de la brecha digital, y que estos pueden ser impartidos tanto en comunidades marginadas, como en espacios de educación básica.

De acuerdo con los talleres descritos en este artículo, se recomienda que la planeación didáctica de un taller de alfabetización digital se desarrolle a partir del modelo propuesto por Semenov et al., y que los pasos considerados dentro de este modelo se interrelacionen entre sí, brindando la posibilidad de un taller más completo y significativo para quienes se encuentran alfabetizándose digitalmente.

De igual forma, se sugiere que durante la evaluación de los talleres de alfabetización digital se considere la adquisición de una o varias de las competencias digitales propuestas por Area y Pessoa, ya que el desarrollo de estas determinará el nivel de alfabetización digital alcanzado tras la intervención.

Por otra parte, a partir de las intervenciones aquí descritas, puede mencionarse que la existencia de programas como UNAMITA ¡Ciérrale a la brecha digital! son de suma importancia para que estudiantes de Educación Superior realicen intervenciones enfocadas en el desarrollo social, cultural y económico dentro de algunas comunidades del país, por lo que se sugiere que se dé un mayor apoyo a este tipo de proyectos. 


\section{REFERENCIAS BIBLIOGRÁFICAS}

Area, M. y Pessoa, T. (2012). De lo sólido a lo líquido: las nuevas alfabetizaciones ante los cambios culturales de la Web 2.0. Revista Científica de Educomunicación, 19(38), 13-20.

Barberá, E. (2006). Portafolio electrónico: desarrollo de competencias profesionales en la red. Revista de Universidad y Sociedad del Conocimiento, 3(2).

Barceló, M. B. (2001). Hacia una Economía del Conocimiento. Madrid, España: ESIC.

Cabero, J. (2004). Reflexiones sobre la brecha digital y la educación. En: F. Soto., y J. Rodríguez. (Ed.), Tecnología, Educación y diversidad: retos y realidades de la inclusión digital (pp. 23-42), Murcia, España: Consejería de Educación y Cultura.

De la UNESCO, I. M. (2005). Hacia las sociedades del conocimiento. Publicaciones Unesco. París.

Fresno, C. (2007). Sobre la necesidad de una alfabetización digital y en información en una sociedad globalizada. ACIMED, 15(4).

Gros, B., y Contreras, D. (2006). La alfabetización digital y el desarrollo de competencias ciudadanas. Revista Iberoamericana de Educación, 42, 103-125.

Gutiérrez, A. (2008). Las TIC en la formación del maestro. "Realfabetización" digital del profesorado. Revista Interuniversitaria de Formación del profesorado, 22(3), 191-206.

Santana, G.A., Castro, L.R., Gutiérrez, J.A., Briones, Y.M., y Mawyin, F.A. (2021). Criterios sobre las tecnologías del aprendizaje y conocimiento (tac) en tiempo de pandemia covid-19. South Florida Journal of Development, 2(2), 1809-1821.

Semenov, A., Pereversev, L., y Bulin-Socolova, H. (2005). Las tecnologías de la información y la comunicación en la enseñanza. Manual para docentes. Montevideo, Uruguay: Trilce. 